\title{
PI3K p110 $\gamma$ overexpression in idiopathic pulmonary fibrosis lung tissue and fibroblast cells: in vitro effects of its inhibition
}

\author{
Enrico Conte ${ }^{1}$, Elisa Gili ${ }^{1}$, Mary Fruciano ${ }^{1}$, Martina Korfei ${ }^{2}$, Evelina Fagone ${ }^{1}$, Maria lemmolo ${ }^{1}$, \\ Debora Lo Furno ${ }^{3}$, Rosario Giuffrida ${ }^{3}$, Nunzio Crimi ${ }^{1}$, Andreas Guenther ${ }^{2}$ and Carlo Vancheri ${ }^{1}$
}

Idiopathic pulmonary fibrosis (IPF) is a progressive fibroproliferative disease whose molecular pathogenesis remains unclear. In a recent paper, we demonstrated a key role for the PI3K pathway in both proliferation and differentiation into myofibroblasts of normal human lung fibroblasts treated with TGF- $\beta$. In this research, we assessed the expression of class I PI3K p110 isoforms in IPF lung tissue as well as in tissue-derived fibroblast cell lines. Moreover, we investigated the in vitro effects of the selective inhibition of p110 isoforms on IPF fibroblast proliferation and fibrogenic activity. IHC was performed on normal and IPF lung tissue. Expression levels of PI3K p110 isoforms were evaluated by western blot and flow cytometry analysis. Fibroblast cell lines were established from both normal and IPF tissue and the effects of selective pharmacological inhibition as well as specific gene silencing by small interfering RNAs were studied in vitro. No significant differences between normal and IPF tissue/tissue-derived fibroblasts were observed for the expression of PI3K p110 $\alpha, \beta$ and $\delta$ isoforms whereas $\mathrm{p} 110 \gamma$ was more greatly expressed in both IPF lung homogenates and ex vivo fibroblast cell lines. Myofibroblasts and bronchiolar basal cells in IPF lungs exhibited strong immunoreactivity for $\mathrm{p} 110 \gamma$. Positive staining for the markers of proliferation proliferating cell nuclear antigen and cyclin D1 was also shown in cells of fibrolastic foci. Furthermore, both $\mathrm{p} 110 \gamma$ pharmacological inhibition and gene silencing were able to significantly inhibit proliferation rate as well as $\alpha$-SMA expression in IPF fibroblasts. Our data suggest that PI3K p110 $\gamma$ isoform may have an important role in the etio-pathology of IPF and can be a specific pharmacological target.

Laboratory Investigation (2013) 93, 566-576; doi:10.1038/labinvest.2013.6; published online 25 February 2013

KEYWORDS: fibroblasts; IPF; lung tissue; myofibroblasts; PI3K p110 $\gamma$

Idiopathic pulmonary fibrosis (IPF) is a relentless interstitial lung disease characterized by fibroblast/myofibroblast accumulation in the alveolar wall and aberrant matrix deposition leading to distortion of the alveolar architecture and the consequent loss of respiratory function. ${ }^{1}$ The typical IPF morphological lesion is the fibroblastic focus, in which fibroblasts/myofibrolasts are embedded in a type I collagenrich matrix. It has been theorized that IPF fibroblasts hold intrinsic alterations of key components of their cellular machinery yet molecular mechanisms differentiating IPF fibroblasts from their normal counterparts remain unknown. In a recent paper, we pinpointed a key role for the PI3K pathway in both proliferation and differentiation into myofibroblasts of lung fibroblasts treated with TGF- $\beta .^{2}$ PI3K signaling has been implicated in a variety of cellular activities including proliferation, survival, adhesion, differentiation, cytoskeletal organization, and so on. ${ }^{3,4}$ The various PI3Ks discovered have been divided into three classes according to their structure and lipid substrate specificity. The most extensively investigated are the class I PI3Ks which, in response to the activation of receptor tyrosine kinases, G protein coupled receptors/cytokine receptors and activated Ras, catalyze the phosphorylation of phosphatidylinositol $(4,5)$-biphosphate (PIP2) to form phosphatidylinositol $(3,4,5)$-triphosphate (PIP3). Prototypical class I PI3K is a dimeric enzyme, consisting of a regulatory and catalytic subunit. The latter

\footnotetext{
${ }^{1}$ Department of Clinical and Molecular Biomedicine, University of Catania, Catania, Italy; ${ }^{2}$ Department of Internal Medicine, University of Giessen Lung Center, JustusLiebig-University Giessen, Giessen, Germany and ${ }^{3}$ Department of Biomedical Sciences, University of Catania, Catania, Italy

Correspondence: Dr E Conte, PhD, Dipartimento di Biomedicina Clinica e Molecolare, Sezione di Medicina Respiratoria, Università di Catania Policlinico Universitario, Via Santa Sofia 78, Catania 95123, Italy.
}

E-mail: econte@unict.it

Received 17 August 2012; revised 10 January 2013; accepted 10 January 2013 
occurs in four isoforms, designated $\mathrm{p} 110 \alpha, \mathrm{p} 110 \beta, \mathrm{p} 110 \gamma$ and $\mathrm{p} 110 \delta$, which are subgrouped into class IA (p110 $\alpha$, p110 $\beta$ and $\mathrm{p} 110 \delta)$ and $\mathrm{IB}(\mathrm{p} 110 \gamma)$. By utilizing pharmacological inhibitors as well as genetic manipulation distinct roles for individual PI3K isoforms have been revealed and distinct kinase functions as well as kinase-independent functions demonstrated. ${ }^{5}$ Although little is currently known about the role of the specific isoforms in airway diseases, targeting specific PI3K isoforms that may be overexpressed or overactive in disease should provide for effective therapeutic treatments. For instance, emerging data from animal models, primary cells and clinical studies in other diseases suggest that specific PI3K/Akt inhibitors can be therapeutically utilized in the treatment of asthma and chronic obstructive pulmonary disease. ${ }^{6-8}$

In this research, we investigated the expression of the class I PI3K isoforms in IPF lung tissue and tissue-derived fibroblasts as well in normal controls. Moreover, ex vivo fibroblast cell lines were used to investigate the effects of isoform-selective inhibitors as well as of specific small interfering RNAs (siRNAs) in fibroblast proliferation and fibrogenic activity.

\section{MATERIALS AND METHODS Lung Tissues}

We utilized IPF tissues from patients with IPF $(n=31$, mean age $49 \pm 11$ years; 13 woman, 17 men; in one case, age and gender were unknown) and controls $(n=15$, mean age $47 \pm 17$ years; 5 women, 8 men; in two cases, age and gender were unknown) from unused organ donors or histologically normal areas of biopsies from surgical operations. Retrospectively, the diagnosis of IPF was reviewed and validated (L Fink, A Guenther) using current American Thoracic Society/European Respiratory Society (ATS/ERS) guidelines, ${ }^{9}$ and patients were included only when current ATS/ERS criteria were met. The study protocol was approved by the ethics committee of the Justus-Liebig-University School of Medicine (no. 31/93, 84/93, 29/01). Informed consent was obtained in written form from each subject for the study protocol.

Lung homogenates were prepared conventionally.

\section{Cell Cultures}

Lung fibroblast cells were derived from IPF lungs (retrospectively diagnosed as above specified) or histologically normal areas of surgical lung specimens from patients undergoing resective surgery. Primary lines were established by using an outgrowth from explants according to the method by Jordana et al ${ }^{10}$ as previously described. ${ }^{11}$ In all the experiments, cell lines were used at a passage earlier than the eight. Before treatment, cells were plated and incubated for $24 \mathrm{~h}$ in serum-free RPMI medium. Afterward, cells were grown for $48 \mathrm{~h}$ in $2 \%$ FBS medium in the absence or presence of PI3K inhibitors. Proliferation rate, AKT phosphorilation, $\alpha$-SMA and collagen production were then evaluated.
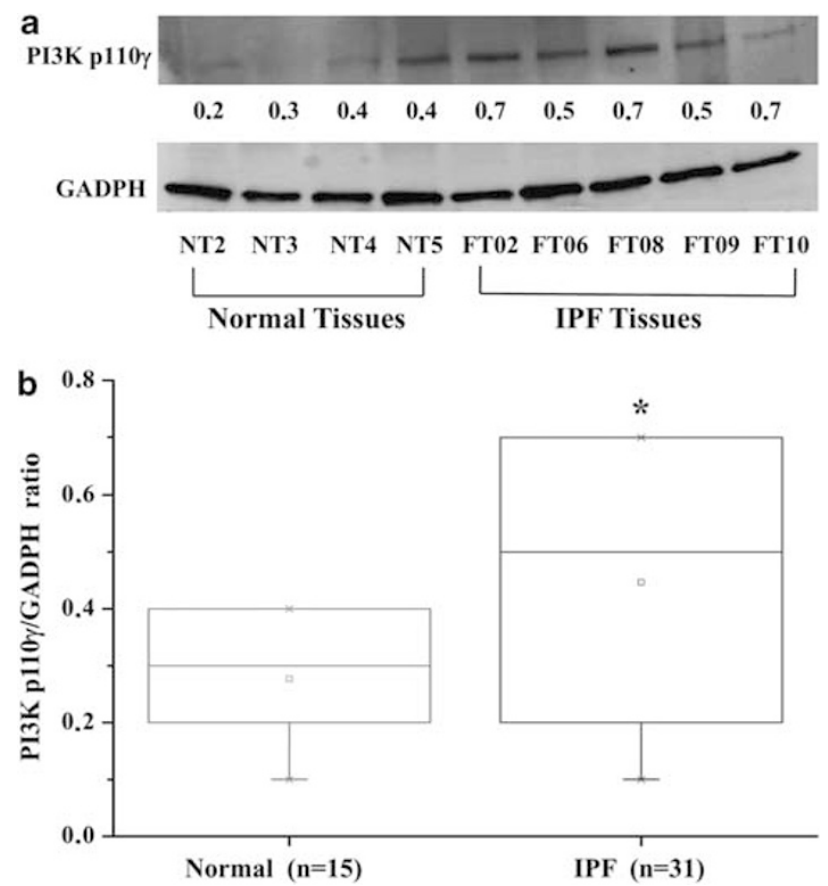

Figure $1 \mathrm{PI} 3 \mathrm{~K}$ p110 $\gamma$ is overexpressed in IPF lung tissue homogenates. Tissues from patients with IPF $(n=31)$ and controls from normal organ donors or histologically normal areas of biopsies from surgical operations $(n=15)$ were utilized for western blot analysis of PI3K p110 isoform expression. (a) Representative p110 $\gamma$ immunoblot of normal and IPF tissues together with housekeeping GAPDH as loading control. Values of target/control gene ratio relative to the pertinent densitometric analysis are indicated. (b) Box and whiskers plot of target/control gene ratio values of all samples revealing an approximately 1.5 -fold median increase in $\mathrm{p} 110 \gamma$ expression in IPF tissues compared with controls. The $P$-value $<0.05$, which indicates a statistically significant difference between relevant groups, is designated with an asterisk.

Transfections with commercially available siRNAs specific for PI3K p110 $\gamma$ as well as with a negative control (Qiagen, Flexi Tube Gene Solution SI00605843, SI02665369 and AllStars Negative Control, respectively) were performed by using Hiperfect Transfection Reagent (Qiagen, Milan, Italy) following the manufacturer's protocol.

\section{PI3K Inhibitors}

Ly294002, AS-252424, TGX-221 and IC87114 were commercially available, YM-024 was kindly provided by Professor Shaun P Jackson (Australian Center for Blood Diseases, Monash University, Melbourne, Australia). TGF- $\beta$ was from Chemicon. All other reagents were from Sigma.

\section{Cell Proliferation}

Cell numbers were determined by counting cells in triplicate on a hemocytometer (Burker chamber) after Trypan blue staining at time 0 (before serum starvation) and after treatments. A mean of four fields was used to calculate the average number of cells. 


\section{Collagen Production}

Total soluble collagen was measured by the Sircol Soluble Collagen Assay (Biocolor, Newtownabbey, UK). The collagen-dye complex was precipitated by centrifugation at $10000 \times g$ for $10 \mathrm{~min}$. The precipitated complex was resuspended in $1 \mathrm{ml}$ alkali reagent. The solution obtained was
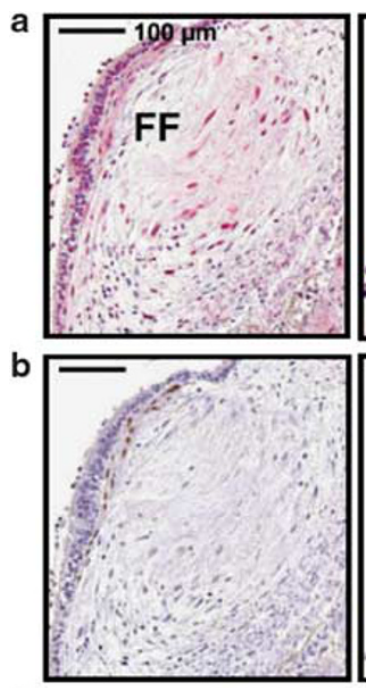

c
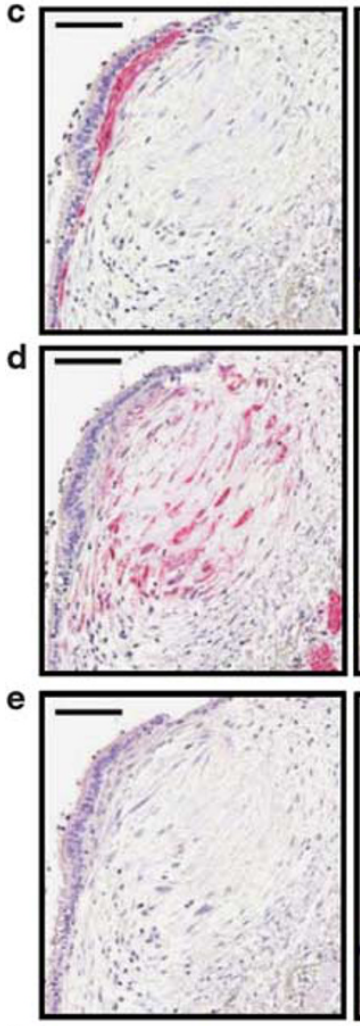

f

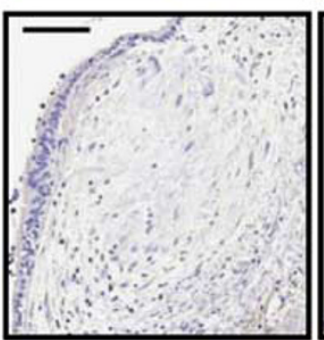

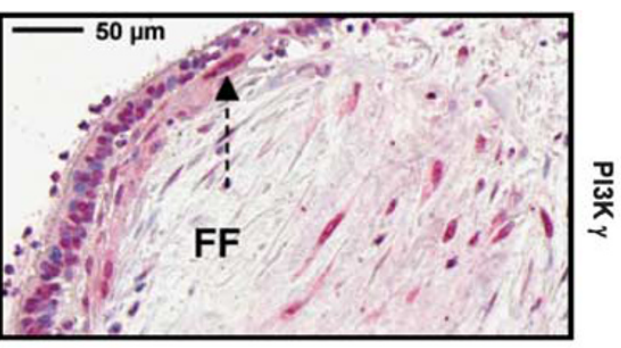
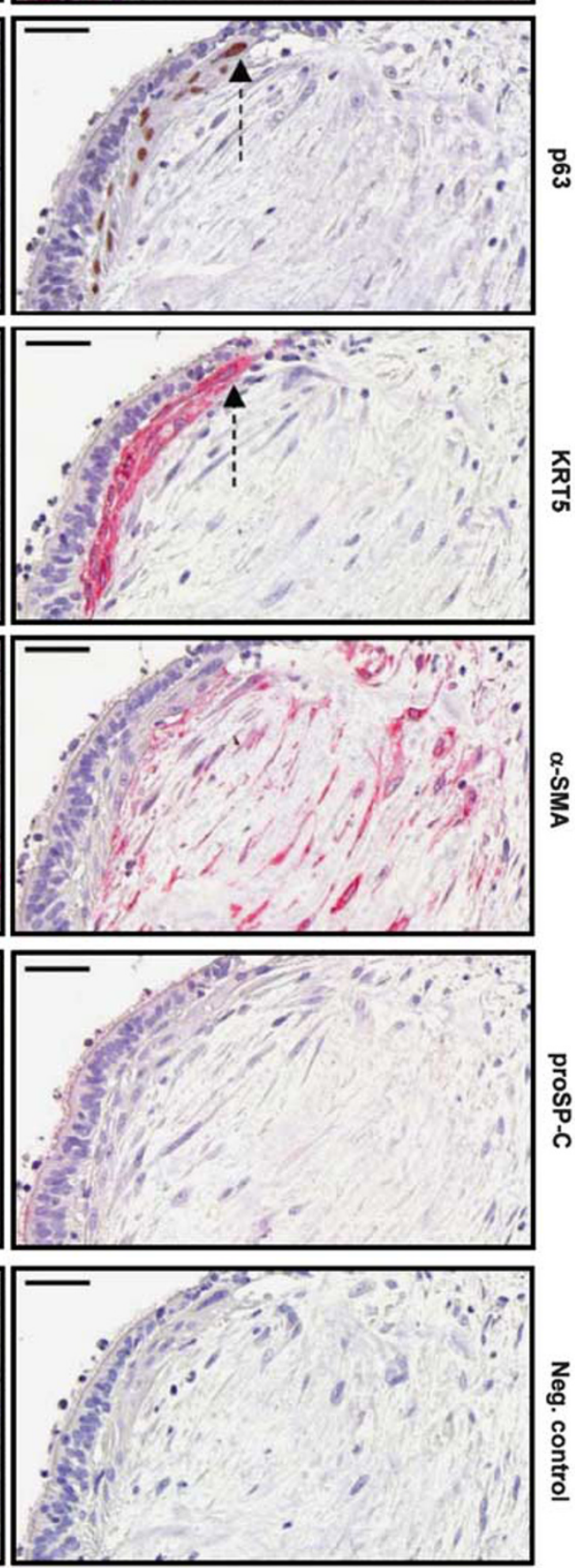

g

怘

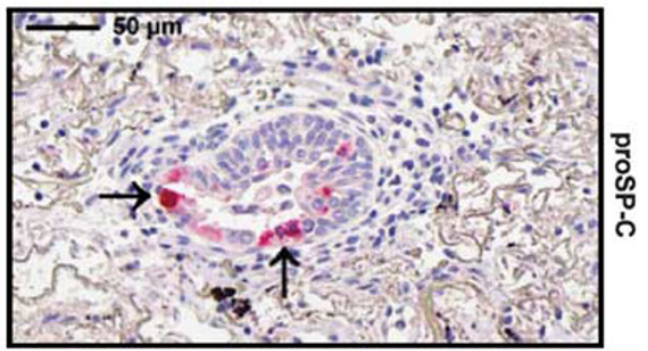

h
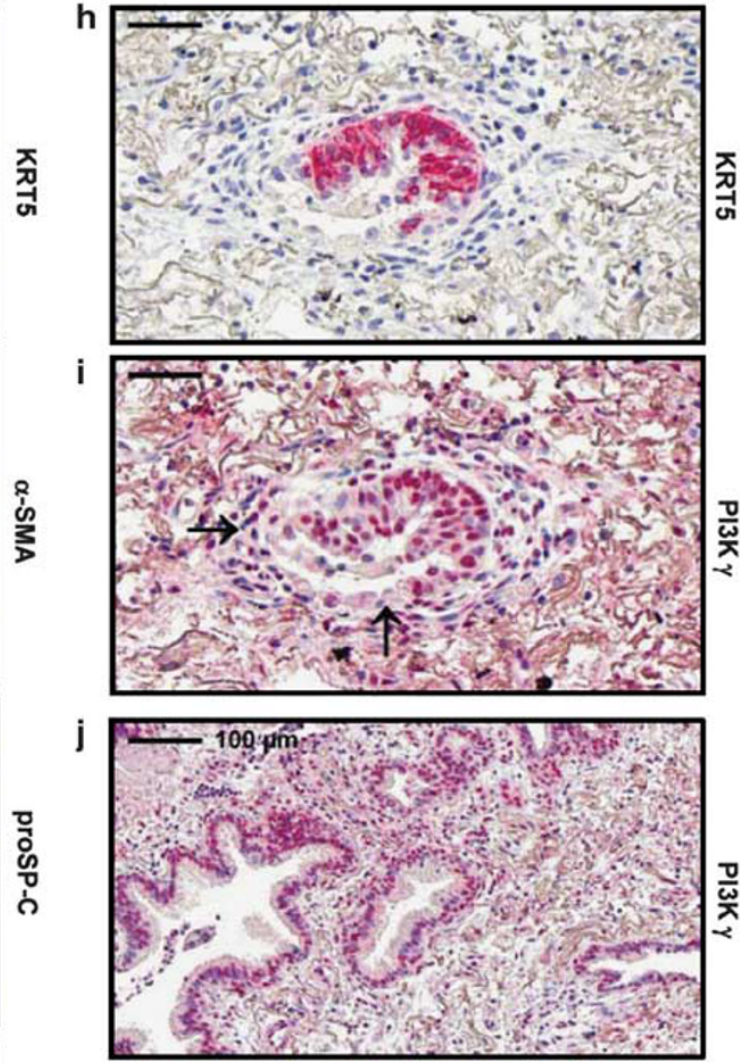

Figure 2 (Continued on next page). 

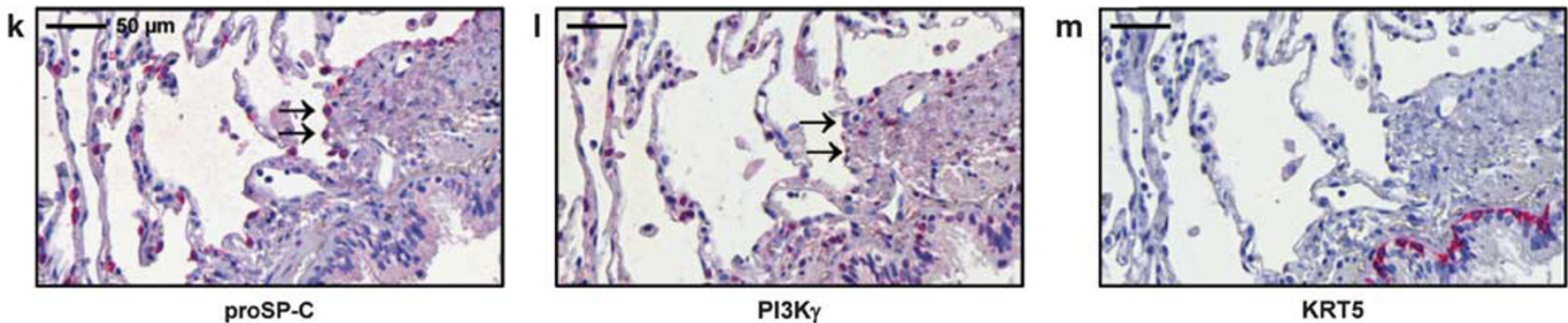

Figure 2 Upregulation of PI3K p110 $\gamma$ in fibroblast foci and bronchiolar epithelial cells in IPF lungs. Representative immunohistochemistry for p110 $\gamma$ $(\mathbf{a}, \mathbf{i}, \mathbf{j})$, p63 (b), cytokeratin-5/KRT5 (c, h), $\alpha$-smooth muscle actin $(\alpha$-SMA) (d) and proSP-C $(\mathbf{e}, \mathbf{g})$ in serial sections of IPF lung tissues $(n=10)$, and for proSP-C (k), p110 $\gamma(\mathbf{I})$ and KRT5 $(\mathbf{m})$ in control lungs $(n=5)$. (a) Strong p110 $\gamma$ antibody staining revealed sustained nuclear and cytoplasmic expression of $\mathrm{p} 110 \gamma$ in the myofibroblasts of fibroblast foci (which were characterized by $\alpha$-SMA expression, (b) as well as in overlying bronchiolar epithelium. Moreover, basal cell sheets, as indicated in serial sections by nuclear p63 and cytoplasmic KRT5 staining (dashed arrows in c and d, respectively), were also positive for $110 \gamma$ (dashed arrows in a). In the overlying epithelium of this area, no type II alveolar epithelial cells (AEClls) were observed as proven by the absence of proSP-C staining (e). In general, the type-II alveolar epithelial cells (AECIIs) of IPF lungs (stained by proSP-C and indicated by arrows in $\mathbf{g}$ ) revealed none or no notable immunoreactivity for $\mathrm{p} 110 \gamma$ antibodies (arrows in i). p110 $\gamma$-positive basal cell layers were also often observed in areas of bronchiolization including abnormalities such as squamous metaplasia (i) or hyperplastic bronchioles (j). In addition, luminal bronchiolar cells in IPF lungs (ciliated and non-ciliated) also expressed p110 $\gamma(\mathbf{a}, \mathbf{j})$. In control lungs, a basal, normal level of p110 $\gamma$ expression was observed in luminal bronchiolar cells, whereas the basal cells of normal bronchioles reveal no notable immunoreactivity (I, $\mathbf{m})$. Similarly, AECIl of control lungs revealed none or faint immunoreactivity to $\mathrm{p} 110 \gamma(\mathbf{k}$, I). Finally, in both IPF- and control lungs pronounced nuclear staining for p110 $\gamma$ was also observed in interstitial inflammatory cells $(\mathbf{i}, \mathbf{j}, \mathbf{l})$. Original magnification of photomicrographs (a-f) (left), and (j): $\times 200$ (bar $=100 \mu$ m); original magnification of photomicrographs (a-f) (right), and $(\mathbf{g}-\mathbf{j}, \mathbf{k}-\mathbf{m}): \times 400$ (bar $=50 \mu \mathrm{m}) . \mathrm{FF}$, fibroblast foci; Neg. control, negative control.

placed in a 96-well flat-bottomed plate and evaluated spectrophotometrically (absorbance, $540 \mathrm{~nm}$ ).

\section{Flow Cytometry Analysis of PI3K p110 $\gamma$ Expression}

The cells were washed, fixed with $2 \%$, paraformaldehyde and permeabilized with Triton 1X (Sigma-Aldrich). Cells were then incubated for 60 min with a monoclonal rabbit anti human-PI3K p110 $\gamma$ (Abcam, Cambridge, UK, ab32089, 1:50). Subsequently, cells were washed once with PBS/BSA $1 \%$ and incubated with Goat $\mathrm{F}(\mathrm{ab}) 2$ Fragment Anti-Mouse IgG (H1L)-FITC (Beckman Coulter, Milano, Italy). Samples were thereafter analyzed by using a Coulter Epics Elite ESP flow cytometer (Coulter Corp., Miami, FL, USA).

\section{Western Blot Analysis}

Lung homogenates or cell lysates were subjected to denaturating SDS gel electrophoresis followed by electroblotting and incubation with monoclonal mouse anti-human $\alpha$-SMA $\mathrm{Ab}$ (1:1000, Dako Cytomation, Denmark), or rabbit anti-human pospho(Ser $\left.{ }^{473}\right)$-Akt (1:500; Cell Signaling Technology, Beverly, MA, USA), monoclonal rabbit anti-human PI3Kp110 $\alpha$ (1:500, Cell Signaling Technology), monoclonal rabbit anti-human PI3Kp110 $\beta$ (1:500, Abcam), monoclonal mouse anti-human PI3Kp110 (1:500, Abcam), monoclonal rabbit anti-human PI3Kp110 $\delta$ (1:500, Abcam) and monoclonal mouse antihuman GAPDH (1:1000, Millipore, Milan, Italy). The membranes were then thoroughly washed and incubated with biotinconjugated anti-mouse or anti-rabbit secondary antibodies (1:5000; Life Technologies) and developed with the Quantum Dot detection system (Invitrogen). The band intensity of exposed film was analyzed by densitometric scanning and quantified using the Image J (NIH, free share) software.

\section{Immunohistochemistry}

Paraffin-embedded lung sections and fibroblast cell cultures were used for immunohistochemical localization of PI3Kp110 $\gamma$ by using monoclonal rabbit anti human-p110 $\gamma$ (Abcam, ab32089, 1:50), or $\alpha$-SMA by rabbit polyclonal ab (Abcam, ab5694, 1:200), cytokeratin-5/KRT5 by mouse monoclonal ab (Abcam, ab7586, 1:200), p63 by rabbit polyclonal (Abcam, ab3239, 1:150), proSP-C by rabbit polyclonal ab (Millipore, AB3786, 1:750), cyclin-D1 by mouse monoclonal ab (Abcam, ab10540 1:50), proliferating cell nuclear antigen (PCNA) by rabbit polyclonal ab (Abcam, ab18197, 1:200) and the ZytoChem Plus Broad Spectrum kit, according to the manufacturer's instructions, following a previously illustrated protocol. ${ }^{12}$

\section{Immunocytochemistry}

After reaching $80 \%$, confluence cells were first washed with PBS, then fixed with $4 \%$ paraformaldheyde in PBS for $30 \mathrm{~min}$ and incubated for $30 \mathrm{~min}$ with a $5 \%$ solution of normal goat serum (Sigma-Aldrich). They were subsequently incubated overnight at $4{ }^{\circ} \mathrm{C}$ with the PI3K p110 primary antibody mentioned above (1:100 dilution). After washing, cells were incubated at room temperature with Cy3-conjugated goat anti-rabbit secondary antibody. Afterward cell were incubated with 4'-6-diamidino-2phenylindole (DAPI, Life Technologies 1:10 000) for nuclear staining, $10 \mathrm{~min}$ in the dark and then washed once. As a control, the specificity of immunostaining was verified by omitting incubation with the primary or secondary antibody. Digital images were acquired using a Leica DMRB fluorescence microscope (Leica Microsystems Srl, Milan, Italy) equipped with a computer-assisted Nikon digital camera (Nital SpA, Turin, Italy). 

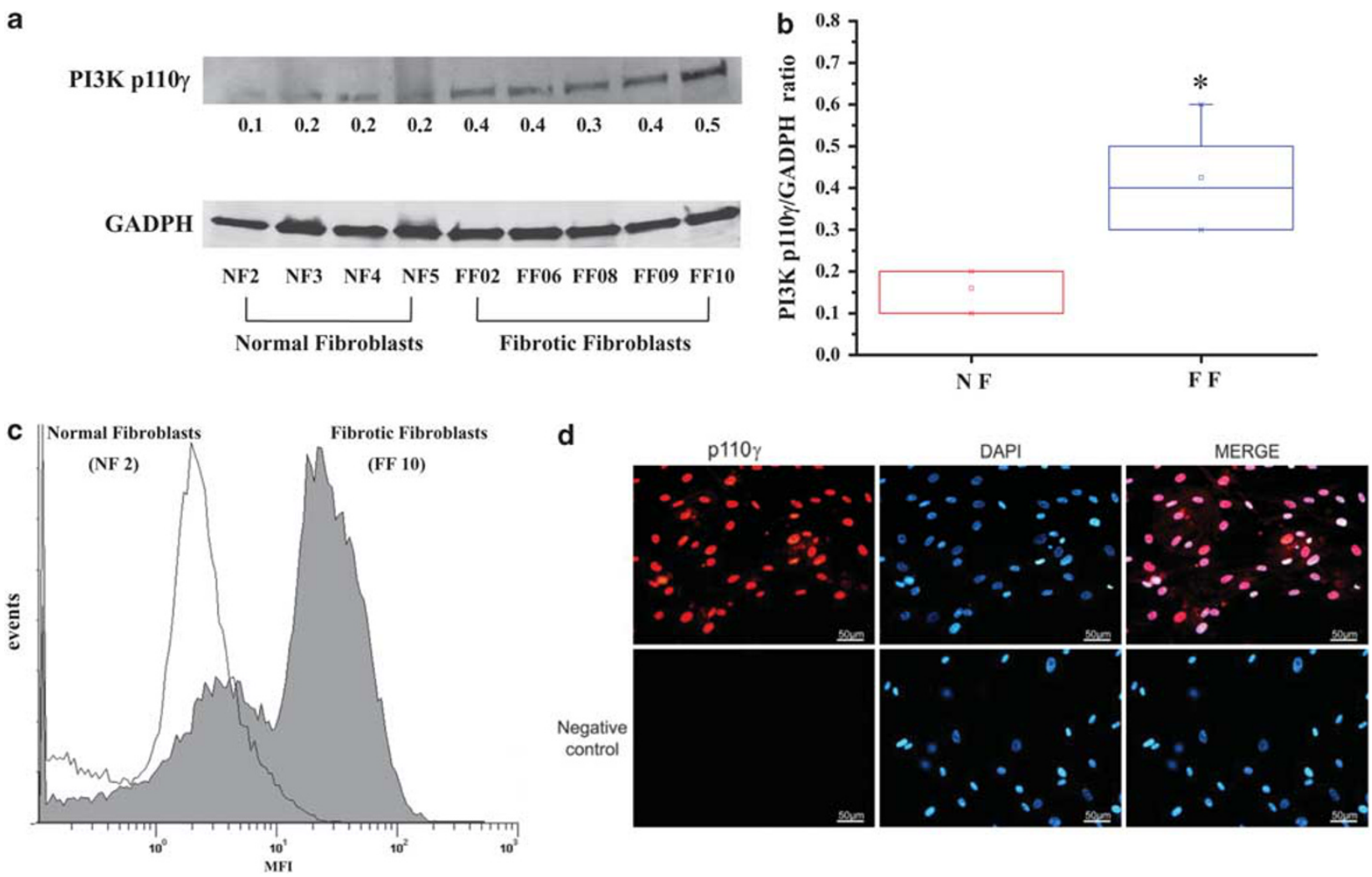

Figure $3 \mathrm{PI} 3 \mathrm{~K}$ p110 $\gamma$ is overexpressed in IPF lung tissue-derived fibroblasts. Western blot and flow cytometry analysis were performed for p110 $\gamma$ expression in fibroblast cell lines established from both normal $(n=8)$ and IPF tissues $(n=15)$. (a) Representative p110 $\gamma$ immunoblot together with housekeeping GAPDH as loading control with indicated values of target/control gene ratio relative to the pertinent densitometric analysis. (b) Box and whiskers plot of ratio values of all samples, both normal (NF) and fibrotic (FF) fibroblasts. The $P$-value $<0.05$ is designated with an asterisk. (c) Flow cytometry plot of p110 expression in a representative normal as well as IPF cell line $(n=3)$. (d) Immunocytochemical analysis in a representative IPF cell line revealing the nuclear localization of $\mathrm{p} 110 \gamma(n=3)$. In the upper panel: slides incubated with monoclonal rabbit anti h-PI3K p110 $\gamma(1: 25)$ and DAPI.

Immunoreactivity was evaluated taking into account the signal-to-noise ratio of immunofluorescence.

\section{Statistical Analysis}

Statistical significance across treatment groups was determined by one-way ANOVA with Tukey's multiple-comparison with Statgraphic Centurion XV software (StatPoint Technologies Inc., Adalta, Italy). A $P$-value $<0.05$, which indicates a statistically significant difference, is designated with a single asterisk.

\section{RESULTS}

\section{PI3K p110 $\gamma$ is Overexpressed in IPF Lung Tissues}

Tissues from patients with IPF $(n=31)$ and controls from organ donors or histologically normal areas of biopsies from surgical operations $(n=15)$ were utilized for western blot analysis of PI3K p110 isoforms' expression. We observed no significant differences between IPF and control tissues in $\mathrm{p} 110 \alpha, \mathrm{p} 110 \beta$ and $\mathrm{p} 110 \delta$ expression (data not shown) whereas $\mathrm{p} 110 \gamma$ appeared overexpressed in IPF lung tissue, as shown in Figure 1. Densitometric quantification of normalized immunoblots revealed an approximate median 1.5-fold increase in $\mathrm{p} 110 \gamma$ content in IPF tissue compared with controls $(P<0.05)$.

Based on this evidence, immunohistochemical analysis for p110 $\gamma$ was performed on serial paraffin-embedded tissue sections of IPF and control lungs. As shown in Figures 2a and $\mathrm{b}$ and in Supplementary Figure 1A, spindle-shaped myofibroblasts in fibroblast foci (FF) exhibited strong nuclear and cytoplasmic immunoreactivity for p110 $\gamma$ in IPF lungs. Moreover, bronchiolar basal cells (characterized by KRT5 and p63 expression) in abnormal bronchiolar structures overlying FF (indicated by dashed arrows in Figures $2 \mathrm{~b}$ and $\mathrm{c}$ ) or in layers of squamous metaplasia (Figures $2 \mathrm{~h}$ and i) or hyperplastic bronchioles (Figure 2j, Supplementary Figure 2) revealed pronounced nuclear expression of PI3K p110 $\gamma$, which was only barely detectable in the basal portion of normal lung tissues (Figures $2 \mathrm{l}$ and $\mathrm{m}$ ) and also in normal bronchioles of IPF lungs (which in some areas have unaffected lung tissue, data not shown). In addition, luminal bronchiolar cells (ciliated and non-ciliated) in IPF lungs revealed also robust nuclear expression of p110 $\gamma$ (Figures $2 \mathrm{a}$ and $\mathrm{j}$ ), whereas only basal expression was observed in bronchioles of donor lung tissues (Figure 2l). As expected, in both categories some interstitial inflammatory 
a

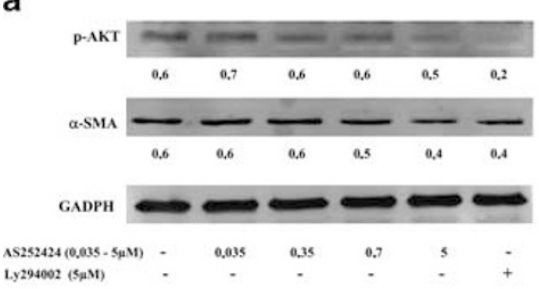

b

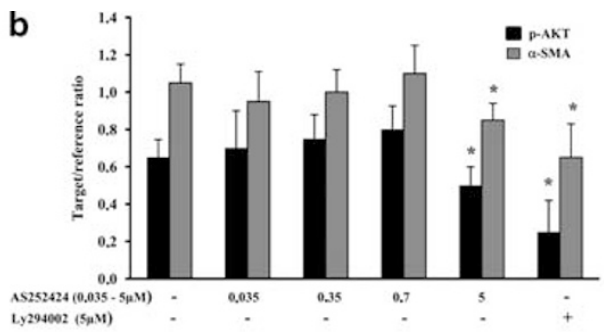

C

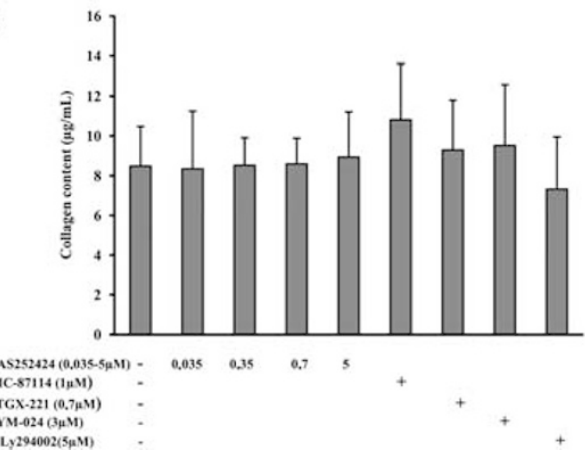

d

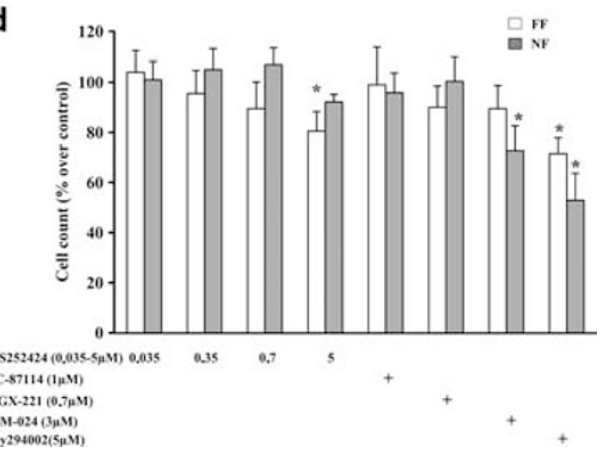

Figure 4 Effects of pharmacological inhibition of class I PI3K p110 isoforms in ex vivo normal and IPF fibroblasts. IPF or normal cells were grown, after initially $24 \mathrm{~h}$ in serum-free medium, for $48 \mathrm{~h}$ in $2 \%$ FBS medium in the absence or presence of PI3K inhibitors. Selective inhibitors of PI3K isoforms: YM-024 for $\mathrm{p} 110 \alpha$, TGX-221 for $\mathrm{p} 110 \beta$, IC87114 for $\mathrm{p} 110 \delta$ and AS-252424 for $\mathrm{p} 110 \gamma$; as well as PI3K pan-inhibitor Ly294002 were used to evaluate the effects on cell proliferation and fibrotic activity in IPF fibroblasts. (a) Representative immunoblot for p110 $\gamma$, p (Ser ${ }^{473}$ )-Akt and GAPDH as loading control in IPF fibroblasts. (b) Mean values of target/control gene ratio relative to the densitometric analysis of blots from three separate experiments with IPF fibroblasts (three different cell lines). (c) Total soluble collagen levels in the supernatants of three separate experiments with IPF fibroblasts. (d) Mean values of cell counting reported as \% over control (ie, cells untreated or treated with pertinent vehicle) in three separate experiments with IPF (white columns, three different cell lines) as well as normal fibroblasts (gray columns, three different cell lines). Statistical significance across treatment groups was determined using one-way ANOVA. A $P$-value $<0.05$ vs relevant untreated controls is designated with an asterisk.

cells (eg, lymphocytes, mast cells, and so on) revealed strong nuclear expression of p $110 \gamma$ (Figures $2 \mathrm{j}$ and $\mathrm{l}$ ). Also vascular smooth muscle cells in IPF- and donor lungs indicated nuclear staining for p110 (data not shown). Of note, type-II alveolar epithelial cells (AECII) in IPF- and donor lungs revealed none or only faint expression of p110 $\gamma$ (indicated by arrows in Figures $2 \mathrm{~g}, \mathrm{k}$ and $\mathrm{l}$ ).

\section{PI3K p110 $\gamma$ is Overexpressed in Fibroblasts Derived from IPF Lung Tissues}

Western blot and flow cytometry analysis was performed for PI3K p110 isoforms in fibroblast cell lines established from both normal and IPF tissues. Paralleling data obtained from tissue observations, no significant differences between normal and IPF fibroblasts were noted for the expression of $\mathrm{p} 110 \alpha, \beta$ and $\delta$ (data not shown) whereas $\mathrm{p} 110 \gamma$ was significantly overexpressed in IPF fibroblasts, as evidenced by western blot analysis (Figures $3 \mathrm{a}$ and b) and flow cytometry (Figure 3c). Immunocytochemical analysis revealed the nuclear localization of p110 $\gamma$ (Figure 3d).

\section{Effects of Pharmacological Inhibition of Class I PI3K p110 Isoforms in Ex Vivo Normal and IPF Fibroblasts}

Cells were grown for $48 \mathrm{~h}$ in $2 \%$ FBS medium in the absence or presence of PI3K inhibitors, after initially $24 \mathrm{~h}$ in serumfree medium. Selective inhibitors of class I PI3K isoforms
(YM-024, TGX-221 and IC87114 for class IA p110 $\alpha$, p110 $\beta$ and $\mathrm{p} 110 \delta$, respectively; AS-252424 for class IB p110 $\gamma$ ) and the PI3K pan-inhibitor Ly294002 as control were used to evaluate any effects on fibrotic activity and cell proliferation of IPF fibroblasts in comparison with normal cells. As shown in Figures 4a and b, significant inhibition of AKT phosphorylation and $\alpha$-SMA expression was achieved in IPF fibroblasts by the p110 $\gamma$ inhibitor AS252424, although only at the highest dose, and as expected by the pan inhibitor Ly294002, whereas collagen content in supernatants was unaffected by all inhibitors of the PI3K p110 isoforms, as shown in panel 4c. At variance with this finding, the p110 inhibitor AS252424 did significantly inhibit cell proliferation in IPF fibroblasts, yet did not in normal fibroblasts where significant proliferation impairment was observed by inhibiting p110 $\alpha$ activity with YM-024 treatment, as shown in Figure 4d.

\section{Effects of the Selective Gene Targeting of $p 110 \gamma$ in Normal and IPF Fibroblasts}

In addition to pharmacological inhibition, specific PI3K p110 $\gamma$ gene silencing was performed by transfection of cells with siRNAs specifically targeting p110 $\gamma$ RNAs. siRNAs with no homology to any known mammalian gene served as a negative controls. Selectivity of p110 $\gamma$ siRNAs was checked by immunoblotting with antibodies against all four p110 

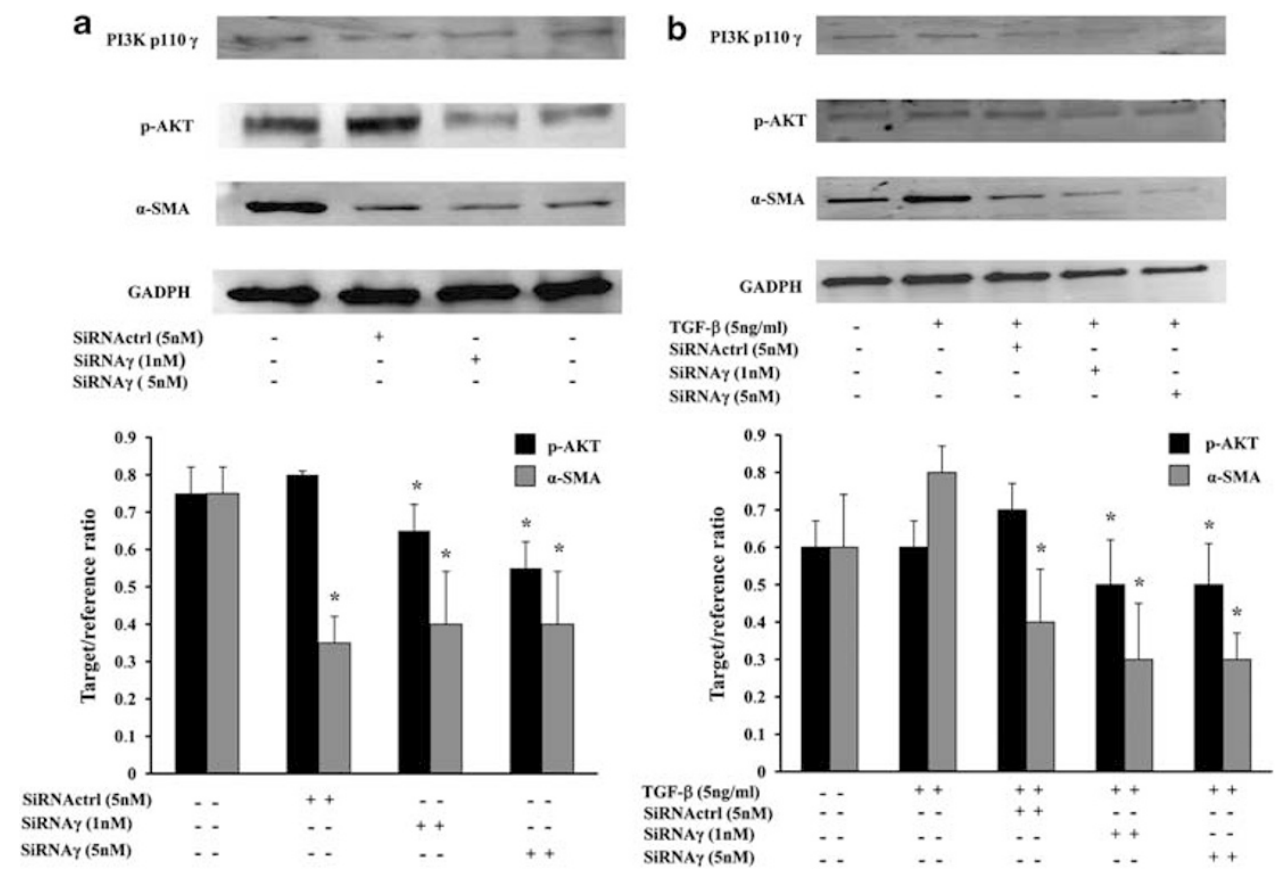

Figure 5 Effects of selective $\mathrm{p} 110 \gamma$ gene targeting in IPF fibroblasts. IPF fibroblasts were plated in $10 \%$ RPMI medium and, after $24 \mathrm{~h}$, incubated for $24 \mathrm{~h}$ in serum-free medium in the absence or presence of commercially available siRNAs specific for PI3K p110 $\gamma$ as well as with a negative control (Qiagen, Flexi Tube Gene Solution SI00605843, SI02665369, 1-5 nM, and AllStars Negative Control, $5 \mathrm{nM}$, respectively). Afterward, cells were grown for $48 \mathrm{~h}$ in $2 \%$ FBS medium in the absence or presence of TGF- $\beta$ and/or siRNAs. (a) Representative immunoblots for p110 $\gamma, \mathrm{p}\left(\mathrm{Ser}{ }^{473}\right.$ )-Akt, $\alpha$-SMA and GAPDH as loading control in IPF fibroblasts (upper panel) as well as mean values of target/control gene ratio relative to the densitometric analysis of blots from three separate experiments with three different cell lines (bottom panel). (b) Representative immunoblots for p110 $\gamma, \mathrm{p}\left(\mathrm{Ser}{ }^{473}\right.$ )-Akt, $\alpha$-SMA and GAPDH as loading control in IPF fibroblasts stimulated with TGF- $\beta$ (upper panel) as well as mean values of target/control gene ratio relative to the pertinent densitometric analysis of blots from three separate experiments with three different cell lines (bottom panel). Statistical significance across treatment groups was determined using one-way ANOVA. A $P$-value $<0.05$ vs untransfected control cells is designated with an asterisk.

isoforms (Supplementary Figure 3). At variance with our previous research, ${ }^{2}$ in this study we used lower concentrations of siRNAs ( $1-5 \mathrm{nmol}$, compared with previous 20 $50 \mathrm{nmol}$ ) although with a more efficient carrier. In Figures 5 and 6 are shown data from at least three separate transfections in three different cell lines of both IPF and normal fibroblasts (unstimulated or stimulated with TGF- $\beta$ ), which obtained comparable results. As indicated by the representative western blots and pertinent densitometric analysis of the overall data relative to both unstimulated and TGF- $\beta$ stimulated cells (Figures 5a and b, respectively), IPF fibroblast transfections with siRNAs targeting p110 $\gamma$ gene expression produced a significant inhibition of AKT-Ser473 phosphorylation compared with the negative controls. The expression of $\alpha$-SMA also was significantly inhibited compared with the untransfected cells but we observed an offtarget effect of the negative control siRNA that significantly inhibited $\alpha$-SMA expression yet leaving AKT-Ser473 phosphorylation unaffected. We thus investigated this off-target effect by using other unrelated negative control siRNAs and we observed the same inhibitory effect (limited to the $\alpha$-SMA expression only). Further experiments are needed to explain this unexpected finding. It is noteworthy that specific p110 $\gamma$ gene targeting significantly inhibited cell proliferation in unstimulated IPF fibroblasts whereas did not in normal fibroblasts, at least at low siRNA concentrations that were used in these experiments (Figure 6a). Moreover, transfections of IPF fibroblasts with siRNAs targeting $\mathrm{p} 110 \gamma$ were able to significantly inhibit cell proliferation also in the presence of TGF- $\beta$ (Figure 6b).

Paralleling data obtained with the pharmacological inhibition, collagen content in supernatants of unstimulated IPF fibroblasts were not affected by specific p110 $\gamma$ gene targeting, as shown in Table 1. Differently, the increase in collagen release induced by TGF- $\beta$ was significantly inhibited by p110 $\gamma$ gene targeting.

\section{Fibroblastic Cells in IPF Lung Tissues Express Markers of Proliferation PCNA and Cyclin D1}

The results obtained from in vitro studies of fibroblasts prompted us to re-analyze the IPF lung tissues in order to consider a putative in situ proliferation activity of fibroblastic cells. A previous study ${ }^{13}$ reported that spindle-shaped cells within fibroblastic foci are positive for PCNA, yet PCNA specificity as marker of proliferation was subsequently questioned. $^{14}$ Therefore, besides PCNA, in this study we 
a

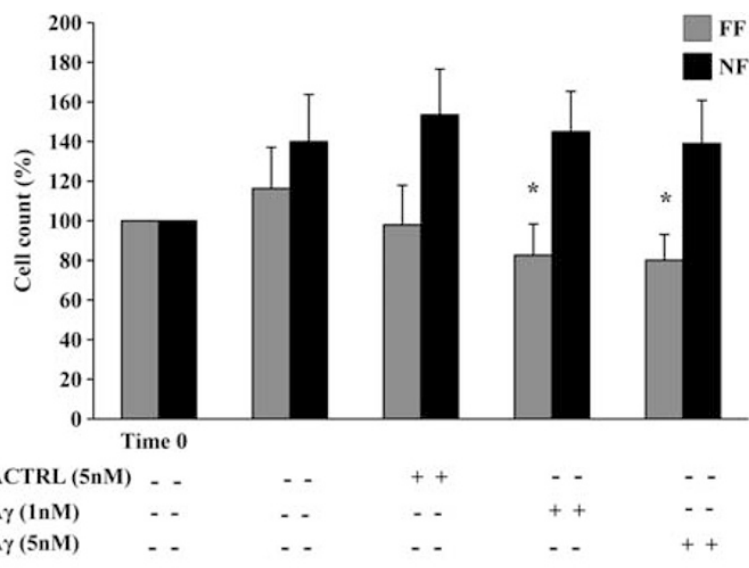

b

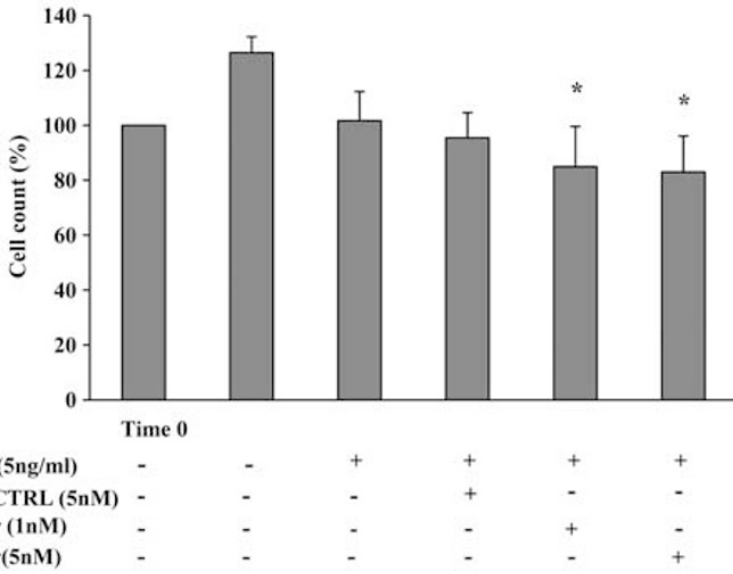

Figure 6 Anti-proliferative effects of $\mathrm{p} 110 \gamma$ gene targeting in normal and IPF fibroblasts. IPF or normal fibroblasts were plated in $10 \%$ RPMI medium and, after $24 \mathrm{~h}$ (time 0 ), incubated for $24 \mathrm{~h}$ in serum-free medium in the absence or presence of siRNAs specific for PI3K p110 $\gamma$ as well as with a negative control. Afterward, cells were grown for $48 \mathrm{~h}$ in $2 \%$ FBS medium in the absence or presence of TGF- $\beta$ and/or siRNAs. (a) Cell counts of at least three separate experiments in three different cell lines of unstimulated IPF and normal fibroblasts, reported as \% of time 0 (range $4.4-8.2 \times 10^{4}$ for normal cells and $5.2-8.6 \times 10^{4}$ for IPF cells, respectively). (b) Cell counts (reported as in a) of three separate experiments in three different cell lines of IPF fibroblasts stimulated with TGF- $\beta$. Statistical significance across treatment groups was determined on raw data using one-way ANOVA. A $P$-value $<0.05$ vs untransfected control cells is designated with an asterisk. evaluated the expression of cyclin D1, a factor strictly required for progression through the G1 phase in the cell cycle.

As shown by results of IHC on serial sections of IPF lung tissues (Figures $7 \mathrm{a}-\mathrm{e}$ ), fibroblastic cells of FF overexpressing $\alpha$-SMA (Figure $7 \mathrm{~b}$ ) and PI3K p110 $($ Figure $7 \mathrm{c}$ ) showed positive staining for antibodies against both cyclin-D1 (Figure 7d) and, as expected, PCNA (Figure 7e).

\section{DISCUSSION}

The molecular mechanisms of IPF pathogenesis are poorly defined and remain a matter of intense discussion and research. In a previous in vitro study, we provided evidence that the PI3K pathway has a major role in both lung fibroblast proliferation and TGF- $\beta$-induced differentiation into myofibroblasts, which are hallmarks of IPF. ${ }^{15}$ In this research, we evaluated the expression of the class I PI3K p110 isoforms in IPF lung homogenates as well as in tissuederived fibroblast cell lines. Our western blot results show that in IPF lung tissues the overall expression of p110 $\alpha$, $\mathrm{p} 110 \beta$ and $\mathrm{p} 110 \delta$ was not significantly different from control tissues whereas $\mathrm{p} 110 \gamma$ was overexpressed. We thus performed immunohistochemical analysis for $\mathrm{p} 110 \gamma$ in IPF tissues and showed that, in addition to the expected interstitial inflammatory cells (eg, lymphocytes, mast cells, and so on), fibroblasts/myofibroblasts exhibited strong nuclear and cytoplasmic immunoreactivity for $\mathrm{p} 110 \gamma$. Interestingly, bronchiolar basal cells in the abnormal bronchiolar structures of IPF lungs also revealed a pronounced nuclear expression of PI3K p110 $\gamma$. These cells seem to share morphological features with those characterized in a previously published paper ${ }^{16}$ indicating that the abnormal proliferation and migration of epithelial basal cells overlying fibroblast foci may have a role in IPF pathological remodeling, leading to bronchiolar colonization/substitution of alveolated parenchyma, leading to lung fibrosis and functional loss. We suggest that the induced overexpression of PI3K p110 $\gamma$ in the basal cells of IPF lungs may cause the exaggerated, proliferative character of this cell type in IPF and thus govern the process of bronchiolization in this disease. Moreover, these aberrant bronchiolar basal cells that are often

Table 1 Effects of selective p110 gene targeting on collagen release in IPF fibroblast cell lines

\begin{tabular}{lccc}
\hline TGF- $\beta(5 \mathrm{ng} / \mathrm{ml})$ & No transfection & SiRNA CTRL $(5 \mathrm{nM})$ & SiRNA $\gamma(1 \mathrm{nM})$ \\
\hline- & $8.06 \pm 1.53 \mu \mathrm{g} / \mathrm{ml}$ & $8.53 \pm 1.12 \mu \mathrm{g} / \mathrm{ml}$ & $8.79 \pm 0.91 \mu \mathrm{g} / \mathrm{ml}$ \\
+ & $14.73 \pm 1.12 \mu \mathrm{g} / \mathrm{ml}$ & $12.64 \pm 3.41 \mu \mathrm{g} / \mathrm{ml}$ & $11.94 \pm 1.22 \mu \mathrm{g} / \mathrm{ml}$
\end{tabular}

Soluble collagen levels in the supernatants of three separate experiments with three different lines of IPF fibroblasts unstimulated or stimulated with TGF- $\beta$ were normalized with the cell number (ie, collagen micrograms $\times$ (untransfected control cells/transfected cells)). 

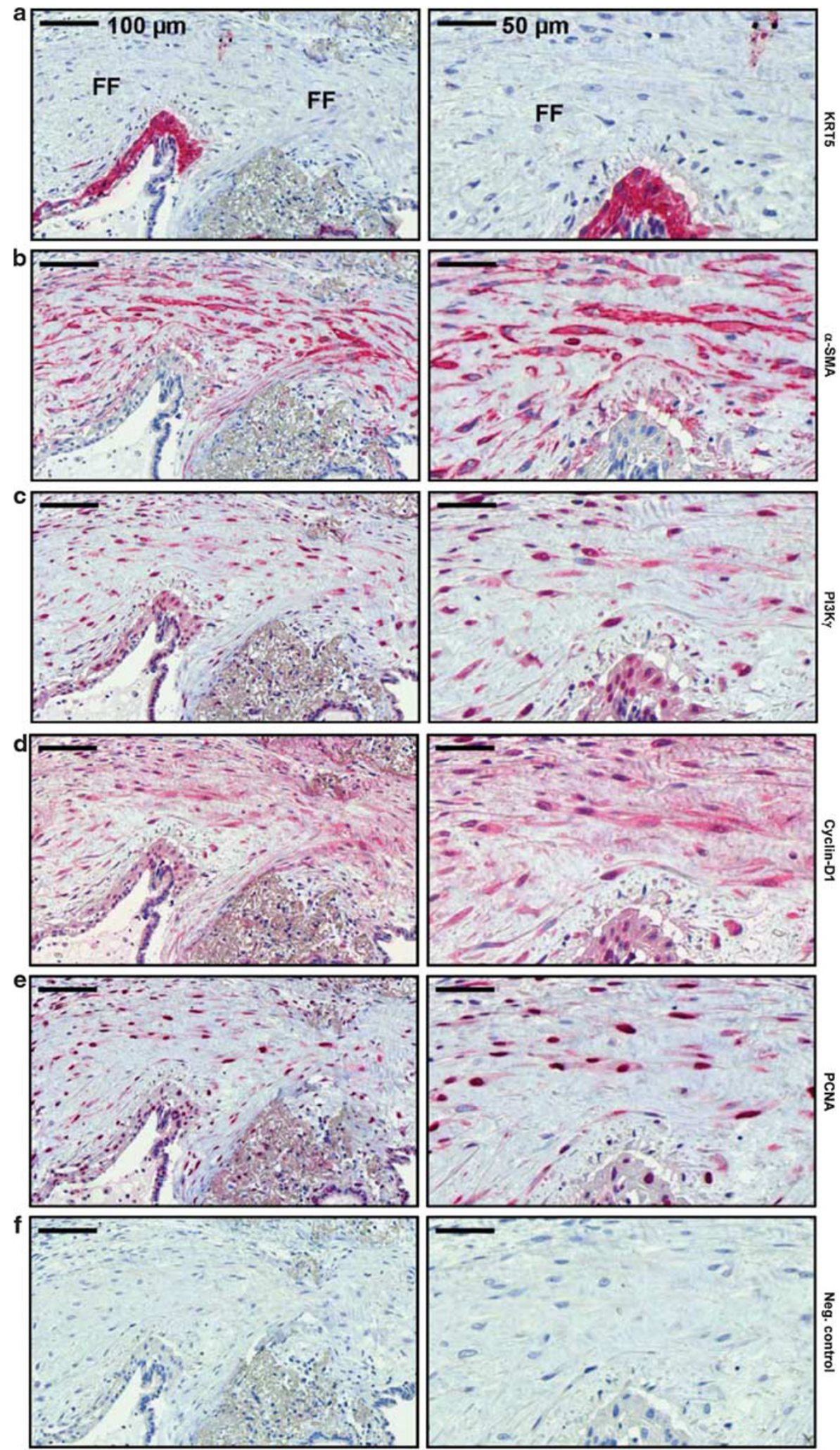

Figure 7 Proliferative activity of $\alpha$-SMA-PI3K p110 $\gamma$-positive cells in fibroblast foci of IPF lungs. Representative immunohistochemistry for cytokeratin-5/ KRT5 (a), $\alpha$-SMA (b), PI3K p110 $\gamma$ (c), cyclin-D1 (d) and PCNA (e) in serial sections of IPF lung tissues. Fibroblastic cells of FF overexpressing $\alpha$-SMA (b) and PI3K p110 $\gamma$ (c) reveal positive staining for antibodies against markers of proliferation cyclin-D1 (d) and PCNA (e). Some bronchiolar basal cells, characterized by KRT5 staining resulted also positive for cyclin-D1 and PCNA. Original magnification of photomicrographs $(\mathbf{a}-\mathbf{f})($ left): $\times 200$ (bar $=100 \mu \mathrm{m})$; original magnification of photomicrographs $(\mathbf{a}-\mathbf{f})$ (right): $\times 400$ (bar $=50 \mu \mathrm{m})$. FF, fibroblast foci; Neg. control, negative control. 
larger may be responsible for the peculiar condition of the abnormal, continuous production of fibrogenic signals in IPF. On the other hand, fibroblast proliferation and fibroblastmyofibroblast differentiation as well as the apoptosis-resistant phenotype of myofibroblasts seem to be mediated by enhanced expression and the action of PI3K p110 $\gamma$ in fibroblast foci themselves. Interestingly, in this study we observed a proliferative activity of PI3K p110 $\gamma$-positive cells as demonstrated by the expression of PCNA and cyclin D1 markers in the spindle-shaped cells within fibroblastic foci visible in serial sections of IPF lung tissues. This finding is consistent with a previously published paper ${ }^{13}$ yet other studies showed a low/very low presence of proliferating cells in fibroblastic foci. ${ }^{14,17}$

On the other hand, increasing evidence suggests that fibroblast proliferation constitutes fundamental ongoing lung injury and activation associated with evolving fibrosis. ${ }^{18-20}$ Moreover, mortality rises in IPF patients who exhibit profusion of fibroblastic foci ${ }^{21,22}$ indicating that the activation of fibroblasts may be a critical regulatory event in the pathogenesis of pulmonary fibrosis. The molecular mechanisms differentiating IPF fibroblasts from their normal counterparts remain unknown. In this research, we isolated fibroblast cell lines from IPF tissues as well as from controls and for the first time we show, in accordance with histological data, significant PI3K p110 $\gamma$ overexpression compared with normal cells. This evidence is in line with a recent observation ${ }^{23}$ showing that fibroblasts isolated from IPF patients display pathological activation of protein kinase B (Akt, downstream to PI3K). Interestingly, we observed a nuclear localization of $\mathrm{p} 110 \gamma$, although the substrate for this kinase in the nucleus remains to be determined. Nevertheless, previous studies in a number of cell types showed the existence of an autonomous nuclear polyphosphoinositide metabolism related to cellular proliferation and differentiation. ${ }^{24,25}$ Moreover, p110 $\gamma$ translocation to the cell nucleus on serum stimulation was experimentally demonstrated. ${ }^{26}$ On the other hand, putative p110 $\gamma$ presence in vascular smooth muscle cell (VSMC) nuclei was correlated with the control of VSMC proliferation and in the pathogenesis of vascular proliferative disorders. ${ }^{27}$

Furthermore, we decided to investigate the in vitro effects of isoform-selective inhibitors of class I PI3Ks on IPF fibroblast proliferation and fibrogenic activity compared with control cells. As in a previous study on normal fibroblasts, ${ }^{2}$ we utilized YM-024, TGX-221 and IC87114 for class IA $\mathrm{p} 110 \alpha, \mathrm{p} 110 \beta$ and $\mathrm{p} 110 \delta$, respectively, and AS-252424 for class IB p $110 \gamma$. Some concerns have retrospectively arisen about the use of this latter as it was very recently shown not to be so specific for $\mathrm{p} 110 \gamma,{ }^{28}$ at least in a mouse model, yet no more selective molecule is available, so notwithstanding we targeted experiments with specific siRNAs as the best way to address the role of p110 $\gamma$ in fibroblast cell proliferation and fibrogenic activity. Our results show that targeting p110\% expression/activity was able to do significantly affect cell proliferation in IPF cultures alone and not in controls. Bearing in mind common features that ourselves and others have previously shown IPF shares with cancer, ${ }^{29,30}$ this finding is particularly interesting considering that in a number of human cancers PI3K p110 $\gamma$ has been shown to be involved in controlling cell proliferation. ${ }^{31-33}$ Also $\alpha$-SMA expression levels were significantly inhibited in IPF fibroblasts by targeting p110 $\gamma$ both pharmacologically and genetically.

Our observations are in line with a previously reported in vivo study ${ }^{34}$ showing that oral administration of a p110 $\gamma$ inhibitor (AS605240) significantly prevented bleomycininduced pulmonary fibrosis in rats also suggesting direct antiproliferative/fibrotic effects on lung fibroblasts, in addition to the stated suppression of inflammatory cell activity.

Taken together, upregulation of PI3K p110 $\gamma$ in myofibroblasts and bronchiolar basal cells in IPF may explain the persistence of myofibroblasts and the abnormal proliferation of basal cells in this fatal lung disease. Although further in vivo investigations are needed, our results indicate that PI3K p110 $\gamma$ might be considered a new target for treating IPF.

Supplementary Information accompanies the paper on the Laboratory Investigation website (http://www.laboratoryinvestigation.org)

\section{ACKNOWLEDGEMENTS}

We are indebted to Dr W Klepetko, Department of Thoracic Surgery, Medical University of Vienna, Austria and to Dr Enrico Potenza, Head of Thoracic Surgery Section of Garibaldi Hospital, Catania Italy, for supplying lung specimens. This research received funding from the European Community's Seventh Framework Program (FP7/2007-2013) under grant agreement no. HEALTH-F2-2007-202224 eurlPFnet.

\section{DISCLOSURE/CONFLICT OF INTEREST}

The authors declare no conflict of interest.

1. American Thoracic Society/European Respiratory Society International Multidisciplinary Consensus Classification of the Idiopathic Interstitial Pneumonias. This joint statement of the American Thoracic Society (ATS), and the European Respiratory Society (ERS) was adopted by the ATS board of directors, June 2001 and by the ERS Executive Committee, June 2001. Am J Respir Crit Care Med 2002;165:277-304.

2. Conte $\mathrm{E}$, Fruciano M, Fagone $\mathrm{E}$, et al. Inhibition of PI3K prevents the proliferation and differentiation of human lung fibroblasts into myofibroblasts: the role of class I P110 isoforms. PLoS One 2011;6: e24663.

3. Cantley LC. The phosphoinositide 3-kinase pathway. Science 2002; 296:1655-1657.

4. Carracedo A, Pandolfi PP. The PTEN-PI3K pathway: of feedbacks and cross-talks. Oncogene 2008;27:5527-5541.

5. Vanhaesebroeck B, Guillermet-Guibert J, Graupera M, et al. The emerging mechanisms of isoform-specific PI3K signalling. Nat Rev Mol Cell Biol 2010;11:329-341.

6. Marwick JA, Chung KF, Adcock IM. Phosphatidylinositol 3-kinase isoforms as targets in respiratory disease. Ther Adv Respir Dis 2010; 4:19-34.

7. Moir LM, Trian T, Ge Q, et al. Phosphatidylinositol 3-kinase isoformspecific effects in airway mesenchymal cell function. J Pharmacol Exp Ther 2011;337:557-566.

8. To $Y$, Ito $K$, Kizawa $Y$, et al. Targeting phosphoinositide-3-kinase-delta with theophylline reverses corticosteroid insensitivity in chronic obstructive pulmonary disease. Am J Respir Crit Care Med 2010;182: 897-904. 
9. Raghu G, Collard HR, Egan JJ, et al. An official ATS/ERS/JRS/ALAT statement: idiopathic pulmonary fibrosis: evidence-based guidelines for diagnosis and management. Am J Respir Crit Care Med 2011; 183:788-824.

10. Jordana M, Schulman J, McSharry C, et al. Heterogeneous proliferative characteristics of human adult lung fibroblast lines and clonally derived fibroblasts from control and fibrotic tissue. Am Rev Respir Dis 1988;137:579-584.

11. Fagone $\mathrm{E}$, Conte $\mathrm{E}$, Gili $\mathrm{E}$, et al. Resveratrol inhibits transforming growth factor-beta-induced proliferation and differentiation of ex vivo human lung fibroblasts into myofibroblasts through ERK/Akt inhibition and PTEN restoration. Exp Lung Res 2011;37:162-174.

12. Korfei M, Schmitt S, Ruppert C, et al. Comparative proteomic analysis of lung tissue from patients with idiopathic pulmonary fibrosis (IPF) and lung transplant donor lungs. J Proteome Res 2011;10:2185-2205.

13. Hagood JS, Prabhakaran P, Kumbla P, et al. Loss of fibroblast Thy-1 expression correlates with lung fibrogenesis. Am J Pathol 2005;167: 365-379.

14. Lepparanta O, Pulkkinen V, Koli K, et al. Transcription factor GATA-6 is expressed in quiescent myofibroblasts in idiopathic pulmonary fibrosis. Am J Respir Cell Mol Biol 2010;42:626-632.

15. Phan SH. The myofibroblast in pulmonary fibrosis. Chest 2002;122(6 Suppl):286S-289SS.

16. Chilosi M, Zamo A, Doglioni C, et al. Migratory marker expression in fibroblast foci of idiopathic pulmonary fibrosis. Respir Res 2006; 7:95.

17. El-Zammar O, Rosenbaum $\mathrm{P}$, Katzenstein AL. Proliferative activity in fibrosing lung diseases: a comparative study of Ki-67 immunoreactivity in diffuse alveolar damage, bronchiolitis obliteransorganizing pneumonia, and usual interstitial pneumonia. Hum Pathol 2009;40:1182-1188.

18. Katzenstein AL, Myers JL. Idiopathic pulmonary fibrosis: clinical relevance of pathologic classification. Am J Respir Crit Care Med 1998;157(4 Pt 1):1301-1315.

19. Kuhn C, McDonald JA. The roles of the myofibroblast in idiopathic pulmonary fibrosis. Ultrastructural and immunohistochemical features of sites of active extracellular matrix synthesis. Am J Pathol 1991;138: 1257-1265.

20. Li $Y$, Jiang $D$, Liang J, et al. Severe lung fibrosis requires an invasive fibroblast phenotype regulated by hyaluronan and CD44. J Exp Med 2011;208:1459-1471.
21. Flaherty KR, Toews GB, Lynch III JP, et al. Steroids in idiopathic pulmonary fibrosis: a prospective assessment of adverse reactions, response to therapy, and survival. Am J Med 2001;110:278-282.

22. King Jr TE, Schwarz MI, Brown K, et al. Idiopathic pulmonary fibrosis: relationship between histopathologic features and mortality. Am J Respir Crit Care Med 2001;164:1025-1032.

23. Xia H, Khalil W, Kahm J, et al. Pathologic caveolin-1 regulation of PTEN in idiopathic pulmonary fibrosis. Am J Pathol 2010;176: 2626-2637.

24. Clarke JH, Letcher AJ, D'santos CS, et al. Inositol lipids are regulated during cell cycle progression in the nuclei of murine erythroleukaemia cells. Biochem J 2001;357(Pt 3):905-910.

25. Divecha N, Clarke JH, Roefs $M$, et al. Nuclear inositides: inconsistent consistencies. Cell Mol Life Sci 2000;57:379-393.

26. Metjian A, Roll RL, Ma AD, et al. Agonists cause nuclear translocation of phosphatidylinositol 3-kinase gamma. A Gbetagamma-dependent pathway that requires the p110gamma amino terminus. J Biol Chem 1999:274:27943-27947.

27. Bacqueville $D$, Deleris $P$, Mendre $C$, et al. Characterization of a $G$ protein-activated phosphoinositide 3-kinase in vascular smooth muscle cell nuclei. J Biol Chem 2001;276:22170-22176.

28. Smith GC, Ong WK, Rewcastle GW, et al. Effects of acutely inhibiting $\mathrm{PI} 3 \mathrm{~K}$ isoforms and mTOR on regulation of glucose metabolism in vivo. Biochem J 2012;442:161-169.

29. Vancheri C, Failla M, Crimi N, et al. Idiopathic pulmonary fibrosis: a disease with similarities and links to cancer biology. Eur Respir J 2010; 35:496-504.

30. Fonar Y, Frank D. FAK and WNT signaling: the meeting of two pathways in cancer and development. Anticancer Agents Med Chem 2011;11:600-606.

31. Dituri F, Mazzocca A, Lupo L, et al. PI3K class IB controls the cell cycle checkpoint promoting cell proliferation in hepatocellular carcinoma. Int J Cancer 2012;130:2505-2513.

32. Edling CE, Selvaggi $F$, Buus $R$, et al. Key role of phosphoinositide 3-kinase class IB in pancreatic cancer. Clin Cancer Res 2010;16:4928-4937.

33. Guerreiro AS, Fattet S, Kulesza DW, et al. A sensitized RNA interference screen identifies a novel role for the PI3K p110gamma isoform in medulloblastoma cell proliferation and chemoresistance. Mol Cancer Res 2011;9:925-935.

34. Wei X, Han J, Chen ZZ, et al. A phosphoinositide 3-kinasegamma inhibitor, AS605240 prevents bleomycin-induced pulmonary fibrosis in rats. Biochem Biophys Res Commun 2010;397:311-317. 